\title{
Many-body and nonequilibrium effects on relaxation oscillations in a quantum-dot microcavity laser
}

Cite as: Appl. Phys. Lett. 97, 111102 (2010); https://doi.org/10.1063/1.3488004

Submitted: 15 February 2010 • Accepted: 19 August 2010 • Published Online: 13 September 2010

Benjamin Lingnau, Kathy Lüdge, Eckehard Schöll, et al.

\section{ARTICLES YOU MAY BE INTERESTED IN}

Theory of relaxation oscillations in semiconductor quantum dot lasers

Applied Physics Letters 89, 101107 (2006); https://doi.org/10.1063/1.2346224

Influence of carrier dynamics on the modulation bandwidth of quantum-dot based nanocavity devices

Applied Physics Letters 97, 211106 (2010); https://doi.org/10.1063/1.3520525

Dynamics of quantum dot photonic crystal lasers

Applied Physics Letters 90, 151102 (2007); https://doi.org/10.1063/1.2720753

四 QBLOX

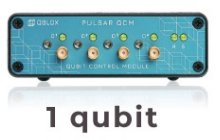

Shorten Setup Time Auto-Calibration More Qubits

Fully-integrated Quantum Control Stacks Ultrastable DC to $18.5 \mathrm{GHz}$ Synchronized $<<1 \mathrm{~ns}$ ultralow noise

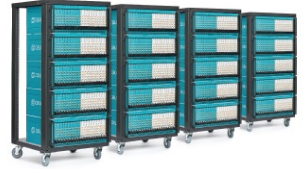

100s qubits

visit our website > 


\title{
Many-body and nonequilibrium effects on relaxation oscillations in a quantum-dot microcavity laser
}

\author{
Benjamin Lingnau, ${ }^{1, a)}$ Kathy Lüdge, ${ }^{1}$ Eckehard Schöll, ${ }^{1}$ and Weng W. Chow ${ }^{2}$ \\ ${ }^{1}$ Institut für Theoretische Physik, Technische Universität Berlin, 10623 Berlin, Germany \\ ${ }^{2}$ Sandia National Laboratories, Albuquerque, New Mexico 87185-1086, USA
}

(Received 15 February 2010; accepted 19 August 2010; published online 13 September 2010)

\begin{abstract}
We investigate many-body and nonequilibrium effects on the dynamical behavior of a quantum-dot laser diode. Simulations, based on the Maxwell-semiconductor-Bloch equations, show strong dependence of the turn-on delay on initial cavity detuning, because of a dynamical shift in the quantum-dot distribution caused by band gap renormalization. Gain switch behavior is found to be insensitive to inhomogeneous broadening, because the balancing between many-body and free-carrier effects inhibits a cavity resonance walk-off. Both the relaxation oscillation damping and frequency are found to increase with decreasing inhomogeneous broadening widths. However, in contrast to bulk and quantum-well lasers, oscillation damping increases less than the frequency.

(C) 2010 American Institute of Physics. [doi:10.1063/1.3488004]
\end{abstract}

The advantages of semiconductor quantum-dot (QD) lasers are being intensively investigated. ${ }^{1}$ Low threshold currents, temperature-insensitive performance, suppression of filamentation, and reduced sensitivity to optical feedback have been theoretically predicted and experimentally demonstrated. ${ }^{2-5}$ Still to be fully realized is the potential for fast dynamical response. ${ }^{6,7}$ Since carrier relaxation is slowed down for discrete energy levels, it is generally agreed that nonequilibrium effects will have greater influence on dynamical behavior in QD lasers. ${ }^{8-10}$ Furthermore, many-body effects were found to influence optical properties. ${ }^{11}$

QD lasers are being modeled using a wide range of approaches. The typical two- or three-variable rate equation model describing total carrier densities, while having the advantage of computational simplicity, cannot systematically account for essential details of the relaxation processes and nonequilibrium situations. ${ }^{12-16}$ In this work, in order to mitigate this deficiency, we consider the momentum resolved population and polarization dynamics, which in an effective relaxation rate approximation allows one to discuss nonequilibrium effects on time scales ranging from subpicoseconds to nanoseconds. Moreover, many-body effects are taken into account at the level of the screened Hartree-Fock approximation. ${ }^{17}$ This approach was further improved by including separate dynamics of electrons and holes in the QDs and in the wetting layer as indicated by quantum kinetic calculations of carrier-carrier scattering rates. ${ }^{18,19}$ Such a model is sufficiently rigorous and comprehensive for investigating QD laser dynamics under most operating conditions.

Discussions in Ref. 17 brought out concerns involving the interplay of nonequilibrium carrier dynamics and manybody effects that can be detrimental to QD microcavity lasers under current modulation conditions. The goal of this paper is to address those concerns, in particular, the extent of how many-body effects, population hole-burning, and inhomogeneous broadening may impact laser design and fabrication.

Central to the model's capability to handle a wide dynamical range is the description of carrier-carrier and carrier-phonon collisions within the effective relaxation rate

${ }^{a)}$ Electronic mail: lingnau@itp.tu-berlin.de. approximation, as opposed to quantum kinetic equations. ${ }^{20}$ Here, $\gamma^{(2)}$ describes the rates at which the charge carrier populations of bulk and quantum-well (QW) states are driven toward a common quasi-Fermi distribution. The rates $\gamma^{(3)}$ include relaxation of bulk, QW and QD states. We label the scattering rates accounting for collisions of two charge carriers with the subscript $\mathrm{c}-\mathrm{c}$, while $\mathrm{c}-\mathrm{p}$ refers to collisions of charge carriers and phonons. In QD lasers, scattering processes for holes are generally faster than those for electrons. ${ }^{18,21}$ This leads to changes in Eqs. (22)-(24) in Ref. 17, describing collision contributions in the Maxwellsemiconductor-Bloch equations, where we have replaced the scattering rates $\gamma_{\mathrm{c}-\mathrm{c}}$ and $\gamma_{\mathrm{c}-\mathrm{p}}$ with the corresponding rates for electrons and holes. Although the basic trends remain unchanged, this improvement noticeably changes the quantitative results. The dynamic variables are the electron and hole densities in the QDs $n_{e}, n_{h}$ and in the wetting layer $w_{e}, w_{h}$ corresponding to $N_{e}^{d}, N_{h}^{d}$ and $N_{e}^{q}, N_{h}^{q}$ in Ref. 17, respectively. Further variables are the laser output power $P$ and the laser frequency detuning $\Delta=\hbar\left(\omega_{c}-\omega\right)$, where $\omega_{c}$ is the cavity mode frequency and $\omega$ is the emission frequency corresponding to the peak of the inhomogeneously broadened QD distribution.

We investigate QD laser dynamical properties by simulating the turn-on of a laser device and its subsequent relaxation oscillations. The QD laser consists of five $\operatorname{In}_{0.2} \mathrm{Ga}_{0.8} \mathrm{As}$ layers, each with a thickness of $d_{L}=3 \mathrm{~nm}$. Embedded in each layer are a density of $N^{\mathrm{QD}}=10^{11} \mathrm{~cm}^{-2} \mathrm{In}_{0.6} \mathrm{Ga}_{0.4} \mathrm{As}$ QDs. The quantum wells are cladded by bulk GaAs layers. The device has a length of $L=50 \mu \mathrm{m}$ and a stripe width of $w_{L}=4 \mu \mathrm{m}$, with a mode confinement factor of $\Gamma=0.20$, which is the fraction of overlap of the laser mode and the QW layers containing the QDs. The energy separations of band edges between bulk and $\mathrm{QW}$ layers are $\Delta E_{C}^{\mathrm{QW}}$ $=155 \mathrm{meV}$ and $\Delta E_{V}^{\mathrm{QW}}=76 \mathrm{meV}$ for the conduction and valence bands, respectively. The QDs have one bound transition with a dipole moment $\mu=0.56 \mathrm{e}_{0} \mathrm{~nm}$. The QD state lies $\Delta E_{e}^{\mathrm{QD}}=52 \mathrm{meV}$ below the $\mathrm{QW}$ conduction band edge for electrons and $\Delta E_{h}^{\mathrm{QD}}=65 \mathrm{meV}$ below the $\mathrm{QW}$ valence band edge for holes, which yields an energy gap of $\hbar \omega_{0}$ $=1.074 \mathrm{eV}$. We describe the QD energy distribution by a 
TABLE I. Numerical parameters used in the simulation unless stated otherwise.

\begin{tabular}{lccc}
\hline \hline \multicolumn{1}{c}{ Symbol } & Value & Symbol & Value \\
\hline$\Delta E_{C}^{\mathrm{QW}}$ & $155 \mathrm{meV}$ & $m_{e}^{*}$ & $0.062 m_{0}$ \\
$\Delta E_{V}^{\mathrm{QW}}$ & $76 \mathrm{meV}$ & $m_{h}^{*}$ & $0.129 m_{0}$ \\
$\Delta E_{e}^{\mathrm{QD}}$ & $52 \mathrm{meV}$ & $N^{\mathrm{QD}}$ & $10^{11} \mathrm{~cm}^{-2}$ \\
$\Delta E_{h}^{\mathrm{QD}}$ & $65 \mathrm{meV}$ & $n^{\text {low }}$ & 3.74 \\
$\gamma_{\mathrm{c}-\mathrm{c}}^{(2)}(e)$ & $20 \mathrm{ps}^{-1}$ & $n^{\text {opt }}$ & 3.34 \\
$\gamma_{\mathrm{c}-\mathrm{p}}^{(2)}(e)$ & $4 \mathrm{ps}^{-1}$ & $\hbar \omega_{0}$ & $1.074 \mathrm{eV}$ \\
$\gamma_{\mathrm{c}-\mathrm{c}}^{(3)}(e)$ & $10 \mathrm{ps}^{-1}$ & $\mu$ & $0.56 e_{0} \mathrm{~nm}$ \\
$\gamma_{\mathrm{c}-\mathrm{p}}^{(3)}(e)$ & $2 \mathrm{ps}^{-1}$ & $\Delta \varepsilon$ & $30 \mathrm{meV}$ \\
$\gamma_{\mathrm{c}-\mathrm{c}}^{(2)}(h)$ & $40 \mathrm{ps}^{-1}$ & $\beta$ & $10^{-4}$ \\
$\gamma_{\mathrm{c}-\mathrm{p}}^{(2)}(h)$ & $8 \mathrm{ps}^{-1}$ & $\Gamma$ & 0.2 \\
$\gamma_{\mathrm{c}-\mathrm{c}}^{(3)}(h)$ & $20 \mathrm{ps}^{-1}$ & $L$ & $50 \mu \mathrm{m}$ \\
$\gamma_{\mathrm{c}-\mathrm{p}}^{(3)}(h)$ & $4 \mathrm{ps}^{-1}$ & $d_{L}$ & $3 \mathrm{~nm}$ \\
$\gamma$ & $10 \mathrm{ps}^{-1}$ & $w_{L}$ & $4 \mu \mathrm{m}$ \\
$\gamma_{\mathrm{rc}}$ & $1 \mathrm{~ns}^{-1}$ & $a_{L}$ & 5 \\
$\gamma_{\mathrm{c}}$ & $1 \mathrm{ps}^{-1}$ & & \\
\hline \hline
\end{tabular}

Gaussian distribution around the mean QD energy $\hbar \omega$ with a standard deviation of $\Delta \varepsilon=30 \mathrm{meV}$ to account for inhomogeneous broadening. The scattering rates along with further parameters used here are listed in Table I, where $\gamma$ is the polarization dephasing rate, $\gamma_{r c}$ is the charge carrier loss rate due to spontaneous recombination or crystal defects, $\gamma_{c}$ is the cavity line width, and $\beta$ is the fraction of spontaneously emitted photons, which contribute to the cavity mode. The effective masses of electrons and holes are $m_{e}^{*}$ and $m_{h}^{*}$, respectively. The active medium refractive indices at low and optical frequencies are given by $n^{\text {low }}$ and $n^{\text {opt }}$, respectively.

Turn-on dynamics of the QD laser is investigated using an injection current $j=7 j_{\text {th }}\left(j_{\text {th }}\right.$ is the laser threshold current density) that is switched-on at $t=0$ with a rise time of $1 \mathrm{ps}$. The solid curve in Fig. 1(a) shows a time trace of the laser output power. It depicts a turn-on delay period followed by a gain-switched peak and strongly damped relaxation oscillations. Compared to the dashed trace that was obtained without many-body effects, there are important quantitative differences arising from dynamical detuning effects. (Note: To accentuate shape differences the dashed curve is shifted in time to match the initial rise of the solid curve. The differ-

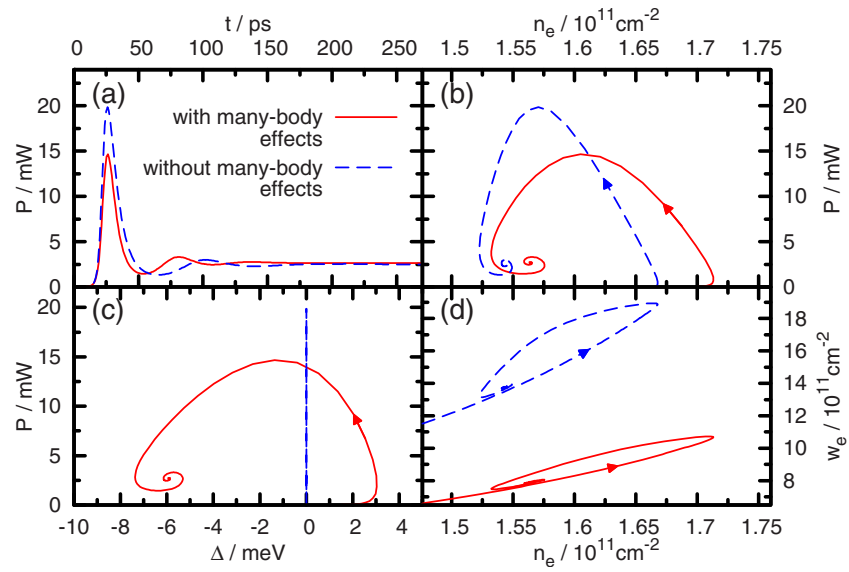

FIG. 1. (Color online) Laser turn-on with (solid) and without (dashed) many-body effects. (a) Superimposed time evolution of optical power after turn-on. (b) Phase portrait of output power vs QD electron density during turn-on. (c) Phase plot of the turn-on showing optical power vs detuning. (d) Turn-on projected onto the $\left(n_{e}, w_{e}\right)$-phase plane.
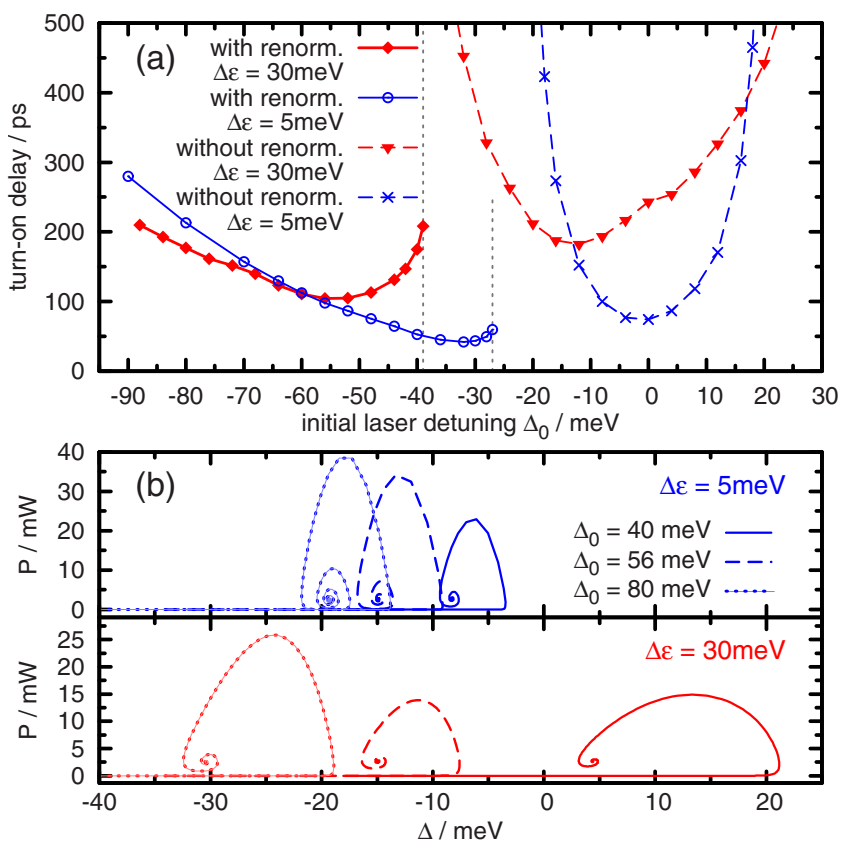

FIG. 2. (Color online) (a) Turn-on delay vs initial cavity detuning with (solid) and without (dashed) many-body effects for inhomogeneous broadening of 5 and $30 \mathrm{meV}$. (b) Phase plot of optical power vs detuning for initial cavity detuning of $-80,-56$, and $-40 \mathrm{meV}$ with $5 \mathrm{meV}$ (upper panel) and $30 \mathrm{meV}$ (lower panel) inhomogeneous broadening.

ence in turn-on delay will be addressed later.) For the manybody result, the initial value of detuning $\Delta$ was chosen so that it reached a value of zero right before actual turn-on. Without many-body effects, a zero detuning was chosen. Figure 1(b) shows the phase space projection of the turn-on process of output power versus QD electron density. Here the QD density $n_{e}$ is the total sum over all electrons in the QD ensemble. It illustrates the internal carrier dynamics with the curve spiraling into the fixed point. The QD electron density at the onset of gain switching as well as at steady state is slightly higher when band gap renormalization is taken into account.

Specifically, the band gap renormalization leads to two effects. At first a dynamical detuning of the laser mode caused by the shifting in the QD distribution. In Fig. 1(c) the laser output power is plotted with respect to the laser detuning. With many-body effects (solid curve) the laser mode is slightly detuned toward lower photon energies (positive detuning) during the initial rise in output power. The detuning then decreases during turn-on and eventually reaches a steady-state value of about $-6 \mathrm{meV}$. Obviously, for the simulation neglecting many-body effects, the detuning remains constant (dashed line). The second effect of the band gap renormalization is depicted in Fig. 1(d). The curves show the turn-on projected onto the $\left(n_{e}, w_{e}\right)$-phase plane, illustrating the QD-QW charge carrier dynamics. Including many-body effects, the absolute QW electron density is lower, while the QD electron density is slightly increased. This is caused by an increase in energy separation between QDs and QW band edge. It shifts the charge carrier distribution toward lower energies, thus leading to a lower electron density in the QW states, which affects relaxation oscillation damping.

In Fig. 2(a) the impact of initial cavity detuning $\Delta_{0}$ on the turn-on delay of the laser is investigated. With $\Delta_{0}>$ $-39 \mathrm{meV}$ for an inhomogeneous broadening of $\Delta \varepsilon$ 

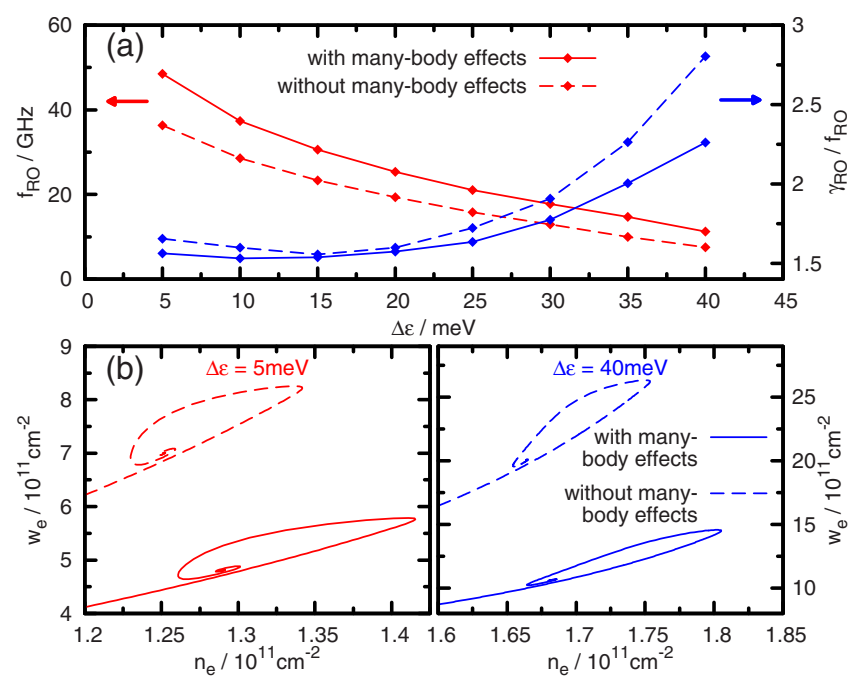

FIG. 3. (Color online) (a) Relaxation oscillation frequency $f_{\text {RO }}$ (left ordinate) and ratio of relaxation oscillation damping coefficient $\gamma_{\mathrm{RO}}$ to frequency (right ordinate) vs inhomogeneous broadening with (solid) and without (dashed) many-body effects. (b) $\left(n_{e}, w_{e}\right)$ phase plots for inhomogeneous broadening of 5 and $40 \mathrm{meV}$, with (solid) and without (dashed) many-body effects.

$=30 \mathrm{meV}\left(\Delta_{0}>-27 \mathrm{meV}\right.$ for $\left.\Delta \varepsilon=5 \mathrm{meV}\right)$, the ensuing band gap renormalization shifts the laser mode too far away from gain maximum to allow lasing. With decreasing $\Delta_{0}$ the turn-on delay passes a minimum and then increases steadily. The dashed curves are obtained by neglecting many-body effects. They show an overall difference in delay times and a more symmetric shape about the delay time minimum, while depending surprisingly more sensitively on $\Delta_{0}$.

At present, substantial effort is directed toward improving QD uniformity to reduce inhomogeneous broadening. It is generally agreed that smaller inhomogeneous broadening contributes directly to overall gain performance. On the other hand, Fig. 2 points to a concern that laser modulation response may suffer because a narrower inhomogeneous width will be less tolerant to detuning changes arising from band gap renormalization. Fortunately, our simulations show that this is not the case because of a balancing between manybody and free-carrier contributions. This is shown in Fig. 2(b), where for 5 and $30 \mathrm{meV}$ inhomogeneous broadening the phase plots of optical power versus detuning for initial cavity detuning of $-80,-56$, and $-40 \mathrm{meV}$ is plotted. Comparing the phase plots, we see that a smaller band gap shift occurs with a narrower inhomogeneous distribution. The reason is that smaller inhomogeneous broadening leads to a higher gain for a given carrier density, or equivalently, a lower threshold carrier density for the same threshold gain. A small carrier density, in turn, gives a smaller renormalization shift, thus preventing the carrier distribution to completely shift away from the lasing mode.

The red curves in Fig. 3(a) show that smaller inhomogeneous broadening leads to higher relaxation oscillation frequency $f_{\mathrm{RO}}$. The reason is that as in $\mathrm{QW}$ and bulk lasers $f_{\mathrm{RO}}$ increases with increasing $j / j_{\text {th }}$. In our case, $j / j_{\text {th }}$ increases with decreasing $\Delta \varepsilon$ because smaller inhomogeneous broadening leads to lower $j_{\text {th }}$ while $j$ is kept fixed. However, in contrast to $\mathrm{QW}$ and bulk lasers, we found that the damping coefficient $\gamma_{\mathrm{RO}}$ increases slower than $f_{\mathrm{RO}}$ increases, as depicted by $\gamma_{\mathrm{RO}} / f_{\mathrm{RO}}$ in Fig. 3(a) (blue curves). This leads to more pronounced relaxation oscillations for narrower QD distributions. The difference in QD relaxation oscillation dynamics can also be seen in the $\left(n_{e}, w_{e}\right)$ phase plots of Fig. 3(b), that show a strongly deformed spiral for $\Delta \varepsilon=40 \mathrm{meV}$ caused by carrier exchange dynamics between QD and QW states.

In conclusion, we apply a nonequilibrium many-body laser model to investigate QD laser dynamics. The study produces two results that impact design and fabrication of QD lasers for injection-current switching and modulation conditions. One is that for applications where control or reproducibility of the turn-on delay is important, it is crucial to have the proper initial cavity detuning with respect to the QD distribution. Nevertheless balancing between many-body and free-carrier effects inhibit a cavity resonance walk-off (from the gain region) and thus inhibit a more drastic sensitivity of gain switch behavior to inhomogeneous broadening. The second result involves relaxation oscillation behavior after turn on, which influences modulation response. We found an overall increase in relaxation oscillation frequency and damping with decreasing inhomogeneous broadening. However, unlike QW and bulk lasers, ${ }^{22}$ the relaxation oscillation damping increases at a slower rate than the relaxation oscillation frequency.

This work was supported by DFG within SFB 787, U.S. Department of Energy under Contract No. DE-AC0494AL85000 and Alexander von Humboldt Foundation.

${ }^{1}$ D. Bimberg, M. Grundmann, and N. N. Ledentsov, Quantum-Dot Heterostructures (Wiley, New York, 1999).

${ }^{2}$ J. Tatebayashi, N. Hatori, H. Kakuma, H. Ebe, H. Sudo, A. Kuramata, Y. Nakata, M. Sugawara, and Y. Arakawa, Electron. Lett. 39, 1130 (2003).

${ }^{3}$ S. Deubert, R. Debusmann, J. P. Reithmaier, and A. Forchel, Electron. Lett. 41, 1125 (2005).

${ }^{4}$ P. M. Smowton, E. J. Pearce, H. C. Schneider, W. W. Chow, and M. Hopkinson, Appl. Phys. Lett. 81, 3251 (2002).

${ }^{5}$ C. Otto, K. Lüdge, and E. Schöll, Phys. Status Solidi B 247, 829 (2010).

${ }^{6}$ S. M. Kim, Y. Wang, M. Keever, and J. S. Harris, IEEE Photonics Technol. Lett. 16, 377 (2004).

${ }^{7}$ S. Fathpour and P. Bhattacharya, J. Phys. D 38, 2103 (2005).

${ }^{8}$ H. Benisty, C. M. Sotomayor-Torres, and C. Weisbuch, Phys. Rev. B 44, 10945 (1991).

${ }^{9}$ M. Sugawara, K. Mukai, and H. Shoji, Appl. Phys. Lett. 71, 2791 (1997).

${ }^{10}$ P. Borri, W. Langbein, J. M. Hvam, F. Heinrichsdorff, M. H. Mao, and D. Bimberg, IEEE J. Sel. Top. Quantum Electron. 6, 544 (2000).

${ }^{11}$ H. C. Schneider, W. W. Chow, and S. W. Koch, Phys. Rev. B 64, 115315 (2001)

${ }^{12}$ F. Adler, M. Geiger, A. Bauknecht, F. Scholz, H. Schweizer, M. H Pilkuhn, B. Ohnesorge, and A. Forchel, J. Appl. Phys. 80, 4019 (1996).

${ }^{13}$ M. Grundmann and D. Bimberg, Phys. Rev. B 55, 9740 (1997).

${ }^{14}$ A. Markus and A. Fiore, Phys. Status Solidi A 201, 338 (2004).

${ }^{15}$ T. Erneux, E. A. Viktorov, and P. Mandel, Phys. Rev. A 76, 023819 (2007).

${ }^{16}$ D. G. Deppe and H. Huang, IEEE J. Quantum Electron. 42, 324 (2006).

${ }^{17}$ W. W. Chow and S. W. Koch, IEEE J. Quantum Electron. 41, 495 (2005).

${ }^{18}$ K. Lüdge and E. Schöll, IEEE J. Quantum Electron. 45, 1396 (2009).

${ }^{19}$ E. Malić, K. J. Ahn, M. J. P. Bormann, P. Hövel, E. Schöll, A. Knorr, M. Kuntz, and D. Bimberg, Appl. Phys. Lett. 89, 101107 (2006).

${ }^{20}$ M. Lorke, F. Jahnke, and W. W. Chow, Appl. Phys. Lett. 90, 051112 (2007).

${ }^{21}$ A. Thränhardt, S. Becker, C. Schlichenmaier, I. Kuznetsova, T. Meier, S. W. Koch, J. Hader, J. V. Moloney, and W. W. Chow, Appl. Phys. Lett. 85, 5526 (2004)

${ }^{22}$ S. Wieczorek, W. W. Chow, L. Chrostowski, and C. J. Chang-Hasnain, IEEE J. Quantum Electron. 42, 552 (2006). 